\title{
Necessidades de saúde de idosos de uma Unidade de Saúde da Família*
}

\author{
Health care needs of the elderly at a Family Health Unit
}

\section{Resumo}

O presente estudo propõe-se a caracterizar as necessidades de saúde entre idosos da área de abrangência de uma Unidade de Saúde da Família (USF). Trata-se de um estudo transversal, realizado com 301 idosos $(65,3 \%)$ da população total da área. $\mathrm{O}$ instrumento de coleta de dados foi construído visando à avaliação multidimensional, que possibilita a identificação das necessidades de saúde no âmbito funcional, social, emocional e ambiental. Constatou-se, entre os entrevistados que 129 (42,9\%) pertencem à faixa etária dos 60 a 69 anos; 186 (61,8\%) são mulheres; $207(68,1 \%)$ são analfabetos ou têm o primeiro grau incompleto; 111 (36,9\%) vivem sem o companheiro; 149 (49,5\%) não realizam qualquer atividade; $218(72,4 \%)$ são considerados pobres ou muito pobres e 161 (53,5\%) vivem apenas da aposentadoria. Em torno de um quarto deles, apresentam sintomatologia de depressão, cognição diminuída e graus de dependência diversos. Os idosos apresentam, ainda, 2,5 diagnósticos/idoso e fazem uso, em média, de 2,9 medicamentos/idoso. Os idosos apresentam necessidades de saúde complexas que demandam planejamento de ações em equipe interdisciplinar.

Universidade Federal de São Paulo, Escola Paulista de Medicina

1 Programa de Pós-graduação em Ciências da Saúde

2 Departamento de Medicina Preventiva. Marília, São Paulo, Brasil

Correspondência/Correspondence

Maria José Sanches Marin

Av. Brigadeiro Eduardo Gomes, 1886 - Jardim Itamarati

17514-000 - Marília, SP, Brasil

E-mail: marnadia@terra.com.br

* Parte do projeto "Os idosos precisam de cuidados especiais: uma proposta para planejamento das ações em uma Unidade de Saúde da Família”, financiado pela Fundação de Amparo a Pesquisa do Estado de São Paulo - FAPESP. Parecer - 06/54664-3.
Palavras-chave:

Necessidades e

Demandas de

Serviços de Saúde.

Idoso Fragilizado.

Saúde da Família.

Estudos Transver-

sais. Avaliação de

Serviços de Saúde.

Determinação de

Necessidades de

Cuidados de Saúde.

Equipe de Assistência ao Paciente.

Programa Saúde da

Família. Saúde do

Idoso. Nível de

Saúde. Avaliação

Geriátrica. 
Abstract

This paper aims to determine the health care needs of individuals aged 60 or more and who live in a Family Health Program area. This cross-sectional study has been carried out with 301 elderly $(65,3 \%)$ of the total population in the area. The data collecting tool was built aiming at multidimensional evaluation that makes possible to identify the following health care needs: functional, social, emotional and environmental. Among the interviewed elderly, 129 (42,9\%) are 60 to 69 years old; 186 (61,8\%) are women; $207(68,1 \%)$ are illiterate or have attended incomplete elementary school; $111(36,9 \%)$ live without the partners; 149 (49,5\%) do not carry out any activity; 218 $(72,4 \%)$ are considered poor or very poor and 161 (53,3\%) live just from their pension. About one fourth of them present depression symptoms, diminished cognition and various dependence degrees. They present 2.5 diagnoses and use an average 2.9 drugs/elderly. The elderly present intense and complex health care needs, which require planning actions with interdisciplinary teams. This is a great challenge to be faced.

Key words: Health Services Needs and Demand. Frail Elderly. Family Health. CrossSectional Studies. Health Services Evaluation. Needs Assessment. Patient Care Team. Family Health Program. Elderly's Health. Level of Health.

Geriatric Assessment.

\section{INTRODUÇÃO}

Atualmente têm sido propostos investimentos que visam a um envelhecimento ativo ${ }^{1}$ e, para isso, é necessária a participação efetiva dos idosos em todas as esferas que envolvem o envelhecimento saudável tanto físico como emocional e social. A promoção da qualidade de vida das pessoas idosas, portanto, é uma necessidade urgente e representa grande desafio para a formulação e implementação das políticas públicas e para a sociedade de maneira geral.

$\mathrm{Na}$ área da saúde, por intermédio da implementação da Estratégia de Saúde de Família (ESF), busca-se um atendimento integral, e em equipe interdisciplinar, ${ }^{2}$ aos idosos - as pessoas que mais procuram o serviço, embora ainda não sejam desenvolvidas ações que atendam especificamente essa parcela da população. ${ }^{3}$ Entendemos que para isso são necessários a prevenção, o tratamento e a reabilitação de alterações no estado de saúde do idoso, o que deve ser conseguido por meio da busca ativa de seus riscos e danos. Tal busca deve fornecer elementos para a elaboração de propostas para o desenvolvimento de ações de saúde visando à melhora das condições de vida.

Por apresentarem características específicas, os idosos requerem uma avaliação de saúde mais cuidadosa, para identificação de problemas subjacentes à queixa. Em seu atendimento, portanto, faz-se necessária a avaliação multidimensional geriátrica abrangente ou avaliação global. ${ }^{4}$

A avaliação multidimensional é uma forma simples de coletar dados capazes de medir consequências, deixando para um plano posterior a investigação das respectivas causas. ${ }^{5}$ Instrumentos de avaliação mul- 
tidimensional possibilitam a identificação do perfil de saúde do idoso, com ênfase nas dimensões que mais comprometem a capacidade funcional da população e, a partir desse perfil, podem-se indicar soluções que transcendem uma linha programática baseada no diagnóstico e terapêutica das doenças crônicas degenerativas. ${ }^{6}$

Considera-se, portanto, relevante desenvolver estudos visando à avaliação multidimensional de idosos que vivem na comunidade, visto que eles apresentam significativos problemas de saúde com vários graus de interferência na autonomia e dependência para as atividades de vida, além de falta de suporte familiar e social que precisam ser evidenciados para o direcionamento de ações de saúde adequadas a cada realidade. Diante do exposto, este estudo propõe-se a caracterizar os idosos, da área de abrangência de uma USF quanto ao perfil sociodemográfico e identificar suas necessidades de saúde por meio da avaliação multidimensional.

\section{METODOLOGIA}

\section{Local e população do estudo}

Trata-se de estudo de caráter descritivo, realizado no ano de 2006, com idosos residentes na área de abrangência de uma Unidade de Saúde da Família (USF), localizada na região central da cidade de Marília. Tal unidade conta atualmente com 1.012 famílias cadastradas, num total de 4.256 pessoas, das quais 461 se encontram na faixa etária acima dos 60 anos, sendo consideradas idosas de acordo com critérios adotados pelo estatuto do idoso.

A população de referência foi constituída pelo universo de idosos residentes na área citada $(n=461)$. A identificação deu-se por meio do cadastro ACS (Agente Comunitário de Saúde). Participaram efetivamente do estudo 301 idosos (65,3\%). Entre os demais, $96(20,8 \%)$ recusaram-se a participar, 61 $(13,2 \%)$ não foram encontrados após a segunda visita e três eram portadores de doenças mentais, encontrando-se sozinhos no domicílio.

\section{Instrumentos de coleta de dados}

Para a coleta de dados foi utilizado um instrumento, elaborado a partir de dados da literatura geriátrica e gerontológica, visando a uma avaliação geriátrica multidimensional, que envolve os aspectos funcionais, emocionais, mentais e risco ambiental, mais especificamente: 1. Características sociodemográficas; 2. Hábitos de vida: fumo, álcool, atividade física e prevenção anual de câncer de útero/mama ou próstata; 3 . Estado cognitivo, estado emocional, grau de dependência, avaliação de presença e graduação da dor. A capacidade cognitiva dos idosos foi avaliada por meio do "Miniexame do estado mental” (MEEM), padronizado para a população brasileira, com a finalidade de caracterizar a presença de prejuízos cognitivos. O teste avalia orientação espacial, atenção, capacidade para realização de cálculos simples, memória recente e linguagem.? 
$\mathrm{Na}$ avaliação do estado emocional, utilizou-se a Escala de Depressão Geriátrica de Yesavage, em versão simplificada com 15 perguntas, que tem demonstrado confiabilidade na prática clínica. ${ }^{8}$ Para avaliação de presença e graduação da dor, utilizou-se uma escala unidimensional - Escala Comportamental (EC) - que se baseia no comportamento da pessoa. Ao comportamento álgico é atribuída uma nota, questionando-se a pessoa quanto à lembrança da dor em função de suas atividades da vida diária. ${ }^{9}$

O grau de dependência foi verificado a partir de uma escala com 12 indicadores de dependência para o idoso, que considera as atividades instrumentais de vida diária ( $f a-$ zer compras, pegar ônibus, administrar as finanças e tomar os medicamentos adequadamente) e as atividades básicas de vida diária (continência, vestir-se, tomar banho, comer pentear-se, ir ao banheiro, passar para cama e sair dela), organizadas hierarquicamente. De acordo com as 12 atividades de vida diária citadas, os idosos são classificados em quatro categorias, a saber: $a$. independente total - idoso capaz de realizar todas as atividades; $b$. dependente leve - idoso que necessita de ajuda para realizar de uma a três atividades de vida diária; c. dependente moderado idoso que necessita de ajuda para realizar de quatro a seis atividades de vida diária e, dependente severo, aquele que necessita de ajuda para realizar sete e ou mais dessas atividades; ${ }^{10}$ 4. Doenças referidas; 5 . Riscos para quedas. Os idosos foram avaliados considerando-se os principais fatores intrínsecos e extrínsecos citados pela literatura. ${ }^{11,12} \mathrm{O}$ equilíbrio e marcha, aspectos essenciais ao se considerarem os riscos de quedas, foram verificados com a utilização do instrumento "Performance Oriented Mobility Assessment POMA", adaptado culturalmente para o Brasil; ${ }^{13}$ 6. Identificação dos medicamentos. Foram considerados aqueles de que o idoso estava fazendo uso no dia da entrevista, incluindo os prescritos e os não prescritos. Para isso, foi solicitado que trouxessem à vista do entrevistador todos os medicamentos em utilização. Os medicamentos foram classificados de acordo com o Anatomical Therapentic Chemical Code (ATC), adotado pela Organização Mundial da Saúde $;^{14} 7$. avaliação do estado nutricional, incluindo Índice de Massa Corporal (IMC), seguindo-se a classificação proposta pela Associação Brasileira para o Estudo da Obesidade e da Síndrome Metabólica (ABESO) e distribuição de gordura corporal, verificada a partir da circunferência da cintura. ${ }^{15}$

\section{Procedimento}

Para realização do estudo, houve autorização do Secretário da Saúde do Município e da equipe de saúde da unidade. Participaram os idosos que, após receberem os esclarecimentos, estavam de acordo e assinaram o Termo de Consentimento Livre e Esclarecido, aprovado pelo Comitê de Ética e Pesquisa com Seres Humanos da Faculdade de Medicina de Marília. A coleta de dados foi realizada no domicílio dos idosos, por estudantes da segunda e terceira séries dos cursos de Enfermagem e Medicina da Faculdade de Medicina de Marília, 
que se dispuseram a tal atividade. Para a utilização do instrumento, eles foram capacitados, contando com um roteiro explicativo a respeito dos itens a serem preenchidos e contaram com acompanhamento e supervisão da pesquisadora responsável.

As informações obtidas foram processadas no programa Epi Info, versão 6.04d, e organizadas de acordo com o percentual, sendo apresentadas em tabelas.

\section{RESULTADOS E DISCUSSÕES}

Os dados do estudo foram obtidos a partir de 65,3\% do universo elegível dos idosos, o que nos permite considerar que há uma grande representatividade dos dados em relação à população total da área. Os resultados apresentados, no entanto, podem apresentar algum viés, em especial referindo-se à classe socioeconômica, pois os idosos que aparentemente apresentavam melhores condições socioeconômicas foram os que mais se recusaram a participar do estudo.

A caracterização sciodemográfica que consta na tabela 1 aponta que quase a metade dos idosos se encontra na faixa etária dos 60 a 69 anos. Além disso, o predomínio do sexo feminino, retratado nos estudos epidemiológicos sobre o processo de envelhecimento, também ocorre no presente estudo. ${ }^{16} \mathrm{Na}$ velhice, atribui-se ao sexo feminino, quando comparado ao sexo masculino, maior vulnerabilidade no estado de saúde em relação a risco de quedas, presença de múltiplas doenças, uso de múltiplos medicamentos, obesidade, pobreza e dependências diversas. ${ }^{17}$ Destaca-se também quanto ao estado marital que 93 (50\%) mulheres vivem sem o companheiro, enquanto apenas $18(15,7 \%)$ homens vivem sós.

A baixa escolaridade dos idosos apresenta-se como um aspecto importante a ser considerado na implementação das ações de saúde, à medida que se constatou que $68,1 \%$ são analfabetos ou tiveram pouco tempo de escolaridade.

Conforme se observa na tabela 1 , o serviço de saúde que a maioria deles (188 - 62,4\%) utiliza é a USF, e 52 (17,3\%), além dos serviços de assistência à saúde do convênio, também recebem atenção dos profissionais $\mathrm{da}$ unidade de saúde da família. Desta forma, amplia-se o papel da equipe frente as necessidade de saúde dessas pessoas.

Como dado complementar das condições de vida dos idosos estudados, tem-se o fato de que mais da metade vive apenas da aposentadoria que, em muitos casos, não passa de um salário mínimo, deixando entrever que eles dispõem de poucos recursos financeiros para uma sobrevivência digna.

Metade dos idosos estudados (49,5\%) não realiza qualquer atividade, o que se torna uma preocupação, pois se sabe que a qualidade de vida depende, em boa medida, da manutenção de alguma atividade regular. 
Tabela 1 - Distribuição dos idosos entrevistados de acordo com a caracterização sociodemográfica. Marília, 2007.

\begin{tabular}{|c|c|c|c|c|c|c|}
\hline \multirow[t]{2}{*}{ Características sociodemograficas } & \multicolumn{2}{|c|}{ Feminino } & \multicolumn{2}{|c|}{ Masculino } & \multicolumn{2}{|c|}{ Total } \\
\hline & $\mathrm{N}^{\circ}$ & $\%$ & $\mathrm{~N}^{\circ}$ & $\%$ & $\mathrm{~N}^{\circ}$ & $\%$ \\
\hline \multicolumn{7}{|l|}{ Faixa etária } \\
\hline $60-69$ anos & 74 & 39,8 & 55 & 47,8 & 129 & 42.9 \\
\hline $70-79$ anos & 76 & 40,8 & 43 & 37,4 & 119 & 39.5 \\
\hline $80-89$ anos & 28 & 15,0 & 17 & 14,8 & 43 & 14.3 \\
\hline$>=90$ anos & 08 & 4,3 & 02 & 1,7 & 10 & 3.3 \\
\hline Total & 186 & 100 & 115 & 100 & 301 & 100 \\
\hline \multicolumn{7}{|l|}{ Escolaridade } \\
\hline Analfabeto & 55 & 29,6 & 22 & 19,1 & 77 & 25 \\
\hline Fundamental incompleto & 84 & 45,1 & 46 & 40,0 & 130 & 43,1 \\
\hline Ensino fundamental & 30 & 16,1 & 25 & 21,7 & 55 & 18,2 \\
\hline Ensino médio & 12 & 6,5 & 16 & 13,9 & 28 & 9,3 \\
\hline Superior & 05 & 2,7 & 06 & 5,2 & 11 & 3,6 \\
\hline Total & 186 & 100 & 115 & 100 & 301 & 100 \\
\hline \multicolumn{7}{|l|}{ Estado Marital } \\
\hline Vive com o companheiro & 93 & 50,0 & 97 & 83,3 & 190 & 63,1 \\
\hline Vive sem o companheiro & 93 & 50,0 & 18 & 15,7 & 111 & 36,9 \\
\hline Total & 186 & 100 & 115 & 100 & 301 & 100 \\
\hline \multicolumn{7}{|l|}{ Profissão e/ou ocupação anterior } \\
\hline Trabalhador braçal & 38 & 20,4 & 47 & 40,9 & 85 & 28,2 \\
\hline Atividade doméstica & 96 & 51,6 & 01 & 0,9 & 97 & 32,2 \\
\hline $\begin{array}{l}\text { Cargo téc./administ/Profissional } \\
\text { liberal }\end{array}$ & 51 & 27,4 & 67 & 58,3 & 118 & 39,2 \\
\hline Nenhuma & 01 & 0,5 & $\infty$ & $\infty 0$ & 01 & 0,3 \\
\hline Tota 1 & 186 & 100 & 115 & 100 & 301 & 100 \\
\hline \multicolumn{7}{|l|}{ Atividade atual principal } \\
\hline Trabalhador braçal & 03 & 1,6 & 06 & 5,2 & 09 & 2,9 \\
\hline Atividade doméstica & 119 & 64,0 & $\infty$ & 00 & 119 & 39,5 \\
\hline $\begin{array}{l}\text { Cargo téc./administ/Profissional } \\
\text { liberal }\end{array}$ & 05 & 2,7 & 19 & 16,5 & 24 & 8,0 \\
\hline Nenhuma & 59 & 31,7 & 90 & 78,3 & 149 & 49,5 \\
\hline Total & 186 & 100 & 115 & 100 & 301 & 100 \\
\hline \multicolumn{7}{|l|}{ Recursos financeiros } \\
\hline Aposentadoria & 101 & 54,3 & 60 & 52,2 & 161 & 53,5 \\
\hline Ajuda de familiares & 04 & 2,2 & 02 & 1,7 & 06 & 2,0 \\
\hline Renda própria & 02 & 2,2 & 09 & 7,9 & 11 & 3,7 \\
\hline Aposentadoria/ajuda de familiares & 61 & 32,8 & 19 & 16,5 & 80 & 26,6 \\
\hline Aposentadoria/ renda própria & 16 & 8,6 & 20 & 17,4 & 36 & 11,9 \\
\hline Outros & 02 & 1,1 & 05 & 4,3 & 07 & 5,5 \\
\hline Tot al & 186 & 100 & 115 & 100 & 301 & 100 \\
\hline \multicolumn{7}{|l|}{ Serviço de saúde que utiliza } \\
\hline USF & 116 & 62,3 & 73 & 63,5 & 188 & 62,4 \\
\hline Convênio & 36 & 19,4 & 23 & 20,0 & 58 & 19,2 \\
\hline Convênio/ USF & 34 & 18,3 & 19 & 16,5 & 52 & 17,3 \\
\hline Total & 186 & 100 & 115 & 100 & 301 & 100 \\
\hline
\end{tabular}

Obs.: O percentual foi calculado, nas duas primeiras colunas, considerando-se o total de idosos por gênero (feminino $\mathrm{n}=186$ e masculino $\mathrm{n}=115)$ e, na última, o total de idosos $(\mathrm{n}=301)$. 
Grau de dependência, estado cognitivo, estado emocional e presença de dor são considerados importantes indicadores da qualidade de vida dos idosos e conhecê-los contribui para o planejamento de políticas públicas cujo foco seja o aumento de anos vividos com saúde pela população (tabela 2).

Os idosos estudados apresentaram graus de dependência diversos. O estado cognitivo estava comprometido em 77 (25,6\%) deles e $102(33,9 \%)$ tinham algum grau de dependência, o que compromete a autonomia para as atividades de vida diária e, consequentemente, sua qualidade de vida.

Estudos realizados em outras comunidades têm mostrado forte relação entre a presença de sintomas de depressão e idade avançada. No presente estudo, $26 \%$ dos ido- sos apresentaram sintomas de depressão, enquanto outros estudos com idosos na comunidade apontam para uma frequência de $31 \% .^{18}$ Ao se avaliar a presença de sintomatologia depressiva com populações de diferentes níveis socioeconômicos (alto, médio e bai$\mathrm{xo})$, foram encontrados os percentuais de $22,6 \%, 19,7 \%$ e $35,1 \%$, respectivamente. ${ }^{19}$ Destaca-se no presente estudo maior quantidade de suspeita de depressão entre as mulheres, $62(33,3 \%)$.

Dos hábitos de vida dos idosos estudados (fumo, álcool, prevenção de câncer de útero/mama ou próstata e atividade física), merece ser considerado o fato de 181 $(60,2 \%)$ dos idosos não realizarem atividade física regular. A influência positiva da atividade física na saúde do idoso vem sendo constantemente demonstrada. ${ }^{20,21}$ 
Tabela 2 - Distribuição dos idosos entrevistados de acordo com estado cognitivo, estado emocional, grau de dependência, presença e graduação da dor e hábitos de vida, segundo sexo. Marília, 2007.

\begin{tabular}{|c|c|c|c|c|c|c|}
\hline \multirow{2}{*}{$\begin{array}{l}\text { Estado cognitivo e emocional, grau de } \\
\text { dependência, dor e hábitos de vida }\end{array}$} & \multicolumn{2}{|c|}{ Feminino } & \multicolumn{2}{|c|}{ Masculino } & \multicolumn{2}{|c|}{ Total } \\
\hline & $\mathrm{N}^{\circ}$ & $\%$ & $\mathrm{~N}^{\circ}$ & $\%$ & $\mathrm{~N}^{\mathrm{o}}$ & $\%$ \\
\hline \multicolumn{7}{|l|}{ Estado cognitivo } \\
\hline Normal & 134 & 72,0 & 90 & 78,3 & 224 & 74,4 \\
\hline Anormal & 52 & 28,0 & 25 & 21,7 & 77 & 25,6 \\
\hline Total & 186 & 100 & 115 & 100 & 301 & 100 \\
\hline \multicolumn{7}{|l|}{ Estado emocional } \\
\hline Sem depressão & 116 & 62,4 & 95 & 82,6 & 211 & 70,1 \\
\hline Suspeita de depressão & 62 & 33,3 & 17 & 14,8 & 79 & 26 \\
\hline Sem condições de avaliar & 08 & 4,3 & 03 & 2,6 & 11 & 3,6 \\
\hline Total & 186 & 100 & 115 & 100 & 301 & 100 \\
\hline \multicolumn{7}{|l|}{ Grau de dependência } \\
\hline Independente & 113 & 60,8 & 86 & 74,8 & 198 & 65,8 \\
\hline Dependente leve & 48 & 25,8 & 15 & 13,0 & 63 & 20,9 \\
\hline Dependente moderado & 16 & 8,6 & 09 & 7,8 & 25 & 8,3 \\
\hline Dependente severo & 09 & 4,8 & 05 & 4,3 & 14 & 4,7 \\
\hline Total & 186 & 100 & 115 & 100 & 301 & 100 \\
\hline \multicolumn{7}{|l|}{ Presença de dor } \\
\hline Nota zero & 89 & 47,8 & 64 & 55,7 & 153 & 50,8 \\
\hline Nota três & 38 & 20,4 & 28 & 24,3 & 66 & 21,9 \\
\hline Nota seis & 30 & 16,1 & 10 & 8,7 & 40 & 13,3 \\
\hline Nota oito & 09 & 4,8 & 10 & 8,7 & 19 & 6,3 \\
\hline Nota dez & 12 & 6,5 & 00 & 00 & 12 & 4,0 \\
\hline Sem condições de avaliar & 08 & 4,3 & 03 & 2,6 & 11 & 3,3 \\
\hline Total & 186 & 100 & 115 & 100 & 301 & 100 \\
\hline \multicolumn{7}{|l|}{ Fumo } \\
\hline Sim & 17 & 9,1 & 18 & 15,7 & 35 & 11,6 \\
\hline Não & 146 & 78,5 & 45 & 39,1 & 191 & 63,4 \\
\hline Ex-fumante & 23 & 12,4 & 52 & 45,2 & 75 & 24,9 \\
\hline Total & 186 & 100 & 115 & 100 & 301 & 100 \\
\hline \multicolumn{7}{|l|}{ Álcool } \\
\hline Sim & 10 & 5,4 & 24 & 20,9 & 34 & 12,3 \\
\hline Não & 174 & 93,5 & 65 & 56,5 & 239 & 79,4 \\
\hline Ex-alcoolista & 02 & 1,1 & 26 & 22,6 & 28 & 9,3 \\
\hline Total & 186 & 100 & 115 & 100 & 301 & 100 \\
\hline \multicolumn{7}{|c|}{ Prevenção de câncer de útero/mama ou próstata anualmente } \\
\hline Sim & 122 & 65,6 & 51 & 44,3 & 173 & 57,5 \\
\hline Não & 64 & 34,4 & 64 & 55,7 & 128 & 42,5 \\
\hline Total & 186 & 100 & 115 & 100 & 301 & 100 \\
\hline \multicolumn{7}{|l|}{ Atividade física } \\
\hline Sim & 79 & 42,5 & 41 & 35,6 & 120 & 39,8 \\
\hline Não & 107 & 57,5 & 74 & 64,4 & 181 & 60,2 \\
\hline Total & 186 & 100 & 115 & 100 & 301 & 100 \\
\hline
\end{tabular}

Obs.: $O$ percentual foi calculado, nas duas primeiras colunas, considerando o total de idosos por gênero (feminino $\mathrm{n}=186$ e masculino $\mathrm{n}=115)$ e, na última, o total de idosos $(\mathrm{n}=301)$. 
Os dados da avaliação nutricional que constam na tabela 3 apontam para a grande proporção de idosos que apresenta sobrepeso e obesidade. Na população brasileira, aponta-se para a quase inexistência de estudos sobre a distribuição do sobrepeso. No entanto, tal índice é considerado importante preditor de morbi-mortalidade Dados de uma população adulta de baixa renda também revelam alta incidência de sobrepeso e obesidade, principalmente nas mulheres. ${ }^{22}$

Com o envelhecimento, além do aumento da gordura corporal, observa-se redistri- buição desse tecido, com acúmulo preferencialmente na região abdominal, o que indica estreita relação com alterações metabólicas e pode desencadear enfermidades cardiovasculares e diabetes mellitus. ${ }^{23}$ Portanto, a verificação da medida da circunferência abdominal tem sido importante preditor do risco para tais doenças. A maioria dos idosos estudados apresenta risco elevado ou muito elevado para o desenvolvimento de doenças associadas à obesidade. Na literatura, faltam estudos epidemiológicos que abordem tal aspecto, em especial referindose à população idosa.

Tabela 3 - Distribuição dos idosos entrevistados de acordo com o estado nutricional. Marília, 2006.

\begin{tabular}{|c|c|c|c|c|c|c|}
\hline \multirow{2}{*}{ Estado Nutricional } & \multicolumn{2}{|c|}{ Feminino } & \multicolumn{2}{|c|}{ Masculino } & \multicolumn{2}{|c|}{ Total } \\
\hline & $\mathrm{N}^{\mathrm{o}}$ & $\%$ & $\mathrm{~N}^{\circ}$ & $\%$ & $\mathrm{~N}^{\mathrm{o}}$ & $\%$ \\
\hline \multicolumn{7}{|l|}{ Índice de Massa corporal (IMC) } \\
\hline IMC menor que 18,5 (abaixo do peso) & 09 & 4,8 & 07 & 6,1 & 16 & 5,3 \\
\hline IMC de 18,5 a 24,9 (Normal) & 53 & 28,5 & 42 & 36,5 & 95 & 31,5 \\
\hline IMC de 25 a 29,9 (sobrepeso) & 80 & 43,0 & 37 & 32,2 & 117 & 38,8 \\
\hline IMC acima de 30 (obesidade) & 31 & 16,7 & 18 & 15,6 & 49 & 16,3 \\
\hline Não foram avaliados & 13 & 7,0 & 11 & 9,6 & 24 & 7,9 \\
\hline Total & 186 & 100 & 115 & 100 & 301 & 100 \\
\hline \multicolumn{7}{|l|}{ Distribuição da gordura corporal } \\
\hline \multicolumn{7}{|l|}{ Circunferência da cintura Mulher } \\
\hline Menor ou igual a 79 & 09 & 4,8 & & & & \\
\hline De 80 a 88 (risco elevado) & 06 & 3,2 & & & & \\
\hline Maior que 89 (risco muito elevado) & 161 & 86,5 & & & & \\
\hline Não foram avaliados & 10 & 5,4 & & & & \\
\hline Total & 186 & 100 & & & & \\
\hline \multicolumn{7}{|l|}{ Circunferência da cintura homem } \\
\hline Menor ou igual a 93 & & & 23 & 20,0 & & \\
\hline De 94 a 102 (risco elevado) & & & 41 & 35,6 & & \\
\hline Maior que 103 (risco muito elevado) & & & 43 & 37,4 & & \\
\hline Não foram avaliados & & & 08 & 7,0 & & \\
\hline Total & & & 115 & 100 & & \\
\hline
\end{tabular}

Obs.: O percentual foi calculado, nas duas primeiras colunas, considerando o total de idosos por gênero (feminino $\mathrm{n}=186$ e masculino $\mathrm{n}=115)$ e, na última, o total de idosos $(\mathrm{n}=301)$. 
Muitos dos idosos estudados vivem em situações de riscos de quedas intrínsecos e extrínsecos, sendo que muitos admitem já terem sofrido quedas. Como muitos deles apresentam diminuição da acuidade visual e auditiva, alterações do equilíbrio e marcha, além de enfrentarem os riscos ambientais, entendemos que tais aspectos devam receber atenção especial por parte da equipe.

Tabela 4 - Distribuição dos idosos entrevistados de acordo com os fatores de risco para quedas. Marília, 2007.

\begin{tabular}{lcccccc}
\hline Fatores de risco para quedas & \multicolumn{2}{c}{ Feminino } & \multicolumn{2}{c}{ Masculino } & \multicolumn{2}{c}{ Total } \\
& N & $\%$ & $N^{\circ}$ & $\%$ & $N^{\circ}$ & $\%$ \\
\hline Número de quedas no último ano & & & & & & \\
\hline Nenhuma & 123 & 66,1 & 92 & 80,0 & 215 & 71,4 \\
De 1 a 2 quedas & 44 & 23,6 & 20 & 17,4 & 64 & 21,2 \\
De 3 a 4 quedas & 11 & 5,9 & 02 & 1,7 & 13 & 4,3 \\
Mais de cinco quedas & 8 & 4,3 & 01 & 0,9 & 09 & 3,0 \\
\hline Sinais e sintomas & & & & & & \\
\hline Diminuição visual & 107 & 57,5 & 67 & 36,2 & 174 & 58,2 \\
Diminuição auditiva & 43 & 23,1 & 35 & 30,4 & 78 & 26,1 \\
Hipotensão ortostática & 00 & 0,0 & 02 & 1,7 & 02 & 0,7 \\
Insônia & 57 & 30,6 & 19 & 16,5 & 76 & 25,4 \\
Urgência/incontinência & 14 & 7,5 & 08 & 7,0 & 22 & 7,4 \\
Problemas nos pés & 47 & 25,3 & 13 & 11,3 & 60 & 20,1 \\
Mobilidade física prejudicada & 66 & 35,5 & 30 & 26,0 & 96 & 32,1 \\
Equilíbrio prejudicado & 113 & 60,8 & 49 & 42,6 & 162 & 53,8 \\
Marcha prejudicada & 103 & 55,4 & 43 & 37,4 & 146 & 48,5 \\
\hline Risco ambiental & & & & & & \\
\hline Piso & 107 & 57,5 & 69 & 60,0 & 176 & 58,5 \\
Escada/degraus & 110 & 59,1 & 74 & 64,3 & 184 & 61,1 \\
Entulhos & 22 & 11,8 & 14 & 12,2 & 36 & 12 \\
Iluminação & 23 & 12,4 & 16 & 13,9 & 39 & 13 \\
Mobília & 34 & 18,3 & 18 & 15,6 & 52 & 17,3 \\
Tapetes & 95 & 51,0 & 56 & 48,7 & 151 & 50,2 \\
\hline Uso de calçado adequado para a idade & & & & & & \\
\hline Sim & 100 & 53,8 & 93 & 80,9 & 193 & 64,1 \\
Não & 86 & 46,2 & 22 & 19,1 & 108 & 39,9 \\
\hline
\end{tabular}

Obs.: O percentual foi calculado, nas duas primeiras colunas, considerando o total de idosos por gênero (feminino $\mathrm{n}=186$ e masculino $\mathrm{n}=115$ ).

Entre as consequências decorrentes doenvelhecimento estão as múltiplas patologias ea ingestão de medicamentos diversos. Os idosos apresentaram em média2,5 diagnósticos referidose, assim como em outros estudos, as doenças cardiovasculares foram aquelas que mais incidiram, seguidas das doenças do sistema osteomuscular e do tecido conjuntivo, além das doenças en- 
dócrinas, nutricionais e metabólicas. A maioria dessas enfermidades, por serem crônicas, demanda alto custo na assistência à saúde, além de propiciar o surgimento de complicações com grande interferência nograu de dependência e qualidade de vida das pessoas.
Como as doenças cardiovasculares lideram as causas de morbi-mortalidade em indivíduos idosos, os medicamentos que atuam nesse sistema são os mais prescritos (Tabela 5$) \cdot{ }^{24}$

Tabela 5 - Classes e subgrupos de medicamentos mais frequentemente utilizados pelos 301 idosos entrevistados. Marilia, 2007.

\begin{tabular}{|c|c|c|}
\hline Classes e subgrupos dos medicamentos & $\mathrm{N}^{\mathrm{o}}$ & $\%$ \\
\hline \multicolumn{3}{|l|}{ Cardiovasculares } \\
\hline Anti-hipertensivo & 180 & 20,4 \\
\hline Diuréticos & 86 & 9,7 \\
\hline Cardiotônico & 11 & 1,2 \\
\hline Atianginosos & 05 & 0,6 \\
\hline Antiarrítimicos & 17 & 1,9 \\
\hline Subtotal & 299 & 33,9 \\
\hline \multicolumn{3}{|l|}{ Sistema Nervoso Central } \\
\hline Hipnóticos, ansiolíticos & 43 & 4,9 \\
\hline Antidepressivos e antimaníacos & 39 & 4,4 \\
\hline Vasodilatador & 30 & 3,4 \\
\hline Anticonvulsivante & 05 & 0,6 \\
\hline Antipsicóticos & 05 & 0,6 \\
\hline Antiparkinonnianos & 05 & 0,6 \\
\hline Subtotal & 138 & 14,4 \\
\hline \multicolumn{3}{|l|}{ Sistema Digestivo e Metabolismo } \\
\hline Insulinas e outros agentes antidiabéticos & 43 & 4,9 \\
\hline Anti-secretores & 30 & 3,4 \\
\hline Antieméticos & 07 & 0,8 \\
\hline Hormônios tiroideanos/antitiroideanos e adjuvantes & 05 & 0,6 \\
\hline Laxativos & 03 & 0,3 \\
\hline Antidiarréicos & 01 & 0,1 \\
\hline Subtotal & 89 & 10,1 \\
\hline \multicolumn{3}{|l|}{ Analgésico, Antiespasmódicos, antiinflamatório } \\
\hline Antiinflamatórios não esteróide & 34 & 3,8 \\
\hline Analgésico & 30 & 3,4 \\
\hline Antiespasmódicos & 10 & 1,2 \\
\hline Antiinflamatórios esteróide & 04 & 0,4 \\
\hline Subtotal & 78 & 8,8 \\
\hline \multicolumn{3}{|l|}{ Sistema Hematopoiético } \\
\hline Antiplaquetário & 46 & 5,2 \\
\hline Anticoagulante e antagonistas & 06 & 0,7 \\
\hline Antianêmico & 02 & 0,2 \\
\hline Subtotal & 54 & 6,1 \\
\hline
\end{tabular}


A população estudada utiliza em média 2,9 medicamentos/idoso. Dados semelhantes foram encontrados em estudo com idosos que frequentam uma Universidade da Terceira Idade, ${ }^{25}$ os quais afirmaram ter ingerido em média 2,3 medicamentos na véspera da entrevista e com idosos atendidos em ambulatório de psicogeriatria, que consumiam três ou mais medicamentos por dia. ${ }^{26}$ No presente estudo, enquanto alguns não consomem nenhum medicamento, encontram-se aqueles que chegam a consumir diariamente de nove a dez, chamando a atenção o fato de mais de um terço dos idosos $105(34,8 \%)$ ingerir quatro ou mais medicamentos.

Outra dificuldade relacionada ao uso de medicamentos é o acesso a eles, uma vez que devido à baixa condição socioeconômica, muitos idosos precisam comprar a medicação, pois esta nem sempre se encontra disponível nas farmácias públicas.

\section{CONCLUSÕES}

No presente estudo constata-se, por meio da avaliação multidimensional, que os idosos que vivem na comunidade apresentam necessidades de saúde complexas, as quais devem ser consideradas ao se proporem intervenções que visem à promoção, prevenção, cura e reabilitação das condições de saúde. Sua identificação pode representar um passo importante na construção do modelo de vigilância da saúde proposto pelo SUS, ${ }^{27}$ cuja busca de consolidação vem-se dando por meio da implementação da ESF.

Os dados do estudo podem subsidiar o planejamento em saúde, devendo ser considerados, além da sua dimensão quantitativa, seu significado para a população envolvida com a situação e a possibilidade de intervir de forma efetiva nos problemas. Além disso, a complexidade da situação descrita exige a articulação da equipe e o desejo de enfrentamento dos problemas de forma interdisciplinar, o que nos parece um grande desafio.

Quando se confrontam com dados as condições de saúde dos idosos, como foi possível pela avaliação multidimensional, torna-se evidente a necessidade de preparo dos profissionais, visando ao desenvolvimento de ações voltadas para as peculiaridades $\mathrm{e}$ complexidade das necessidades apresentadas por eles. $\mathrm{Na}$ segunda fase do estudo, os dados que ora apresentamos serão discutidos com a equipe da unidade, com vistas ao planejamento das atividades.

No planejamento das ações de saúde para idosos que vivem na comunidade há de se considerar, ainda, o conceito de "envelhecimento bem-sucedido", que engloba três domínios multidimensionais: evitar doenças e incapacidades, manter uma alta função física e cognitiva e engajar-se de forma sustentada em atividades sociais e produtivas. ${ }^{28}$ 


\section{REFERÊNCIAS}

1. World Health Organization.

Envelhecimento ativo: uma política de saúde. Brasília: Organização PanAmericana da Saúde; 2005.

2. Merhy EE. E daí surge o PSF como uma continuidade e um aperfeiçoamento do PACS. Interface 2001; 5(9): 147-9.

3. Silva JB Jr, Gomes FBC, Cezário AC, Moura L. Doenças e agravos nãotransmissíveis: bases epidemiológicas. In: Rouquayrol MZ, Almeida Filho N. Epidemiologia \& saúde. 6. ed. Rio de Janeiro: Medsi; 2003. p. 289-311.

4. Pacheco RO, Santos SSC. Avaliação global de idosos em unidade de PSF. Textos sobre Envelhecimento 2004; 7(2): 45-61.

5. Menezes AK. Avaliação funcional do idoso versus avaliação clínica tradicional. In: Sociedade Brasileira de Geriatria e Gerontologia - RJ. Caminhos do envelhecer. Rio de Janeiro(RJ): Revinter; 1994. p. 63-70.

6. Ramos LR. Fatores determinantes do envelhecimento saudável em idosos residentes em centro urbano: Projeto Epidoso, São Paulo. Cad Saude Publica 2003; 19(3): 793-8.

7. Brucki SMD, Nitrini R, Caramelli P, Bertolucci, PH, Okamoto IH. Sugestões para o uso do mini-exame do estado mental no Brasil. Arq Neuropsiquiatr 2003; 61:777-81.

8. Almeida OP, Almeida SA. Confiabilidade da versão brasileira da Escala de Depressão em Geriatria (GDS) versão reduzida. Arq Neuropsiquiatr 1999; 57 : 421-6.

9. Andrade FA, Pereira LV, Sousa, FAEF. Mensuração da dor no idoso: uma revisão. Rev Lat Am Enfermagem 2006; 14(2): 271-6.

10. Ramos LR, Perracini MR, Rosa TE, Kalache A. Significance and management of disability amoung urban elderly residents in Brazil. J Cross Cult Gerontol 1993; 8: 313-23.

11. North American Diagnosis Association NANDA. Diagnósticos de enfermagem da NANDA: definições e classificação 2001-2002. Porto Alegre: Artmed; 2004.

12. PEQUI: Portal Equilíbio e Quedas em Idosos. [homepage na Internet]. São Paulo: FAPESP. Perracini MR. Prevenção e manejo de quedas no idoso. [acesso em: 30 jun 2005]. Disponível em: URL: http:// pequi.incubadora.fapesp.br/portal/ quedas/quedas.pdf.

13. Gomes GC. Tradução, adaptação transcultural e exame das propriedades de medida da escala "Performance-oriented mobility assessment" (POMA) para uma amostragem de idosos brasileiros institucionalizados [dissertação]. Campinas: Universidade Estadual de Campinas; 2003.

14. World Health Organization. The safety of medicines in public health programmes: pharmacovigilance na essential tool. Geneve: WHO; 2006. [acesso em: 11 nov 2007]. Disponível em: URL: http:// www.who.int/medicines/areas/ quality_safety/safety_efficacy/ Pharmacovigilance_B.pdf

15. Associação Brasileira de Obesidade. Métodos de avaliação de obesidade e alguns dados epidemiológicos. São Paulo (SP). [acesso em: 07 fev 2008]. Disponível em: URL: http://www.abeso.org.br/ revista/revista11/metodos.htm 
16. Fundação Sistema Estadual de Análise de Dados. Esperança de vida aumenta e diferença entre gêneros diminui: queda de homicídios em jovens poupa vidas e explica avanço masculino. [monografia na Internet]. São Paulo; c2007. [acesso em: 31 mar 2007]. Disponível em: URL: http:// www.seade.gov.br/produtos/espvida/ espvida_jan2006.pdf

17. Camargos MCS, Perpétuo IHO, Machado CJ. Expectativa de vida com incapacidade funcional em idosos em São Paulo. Rev Panam Salud Publica 2005; 17: 379-86.

18. Oliveira DAAP, Gomes L, Oliveira, RF. Prevalência de depressão em idosos que frequentam centros de convivência. Rev Saúde Pública 2006; 40: 734-6.

19. Veras RP, Murphy E. The mental health of older people in Rio de Janeiro. Int J Geriatr Psychiatry 1994; 9: 285-95.

20. Santarém JM. Promoção da saúde: a importância da atividade física. 1998. [acesso em 5 fev 2008]. Disponível em: URL: http://www.saudetotal.com/ artigos/atividadefisica/idoso.asp

21. Nóbrega ACL, Freitas EV, Oliveira MAB, Leitão MB, Lazzoli JK, Nahas RM, et al. Posicionamento oficial da Sociedade Brasileira de Medicina do Esporte e da Sociedade Brasileira de Geriatria e
Gerontologia: atividade física e saúde no idoso. Revista brasileira de medicina do esporte 1999; 5: 207-11.

22. Anjos LA, Silva DO, Serrão, SA, Silva CVC. Vigilância nutricional em adultos: experiência de uma unidade de saúde atendendo população favelada. Cad Saúde Pública 1992; 8: 50-6.

23. Sampaio LR. Avaliação nutricional e envelhecimento. Rev Nutr 2004; 17: 507-14

24. Ali Raza J, Movahed A. Use of cardiovascular medications in the elderly. Int J Cardiol 2002; 85: 203-15.

25. Sayd JD, Figueiredo MC, Vaena MLHT. Automedicação na população idosa do núcleo de atenção ao idoso da UnATIUERJ. Textos sobre Envelhecimento 2000; 3(3): 21-34.

26. Almeida OP, Ratto L, Garrido R, Tamai S. Fatores preditores e consequências clínicas do uso de múltiplas medicações entre idosos atendidos em um serviço ambulatorial de saúde mental. Rev Bras Psiquiatr 1999; 21(3): 152-7.

27. Teixeira CF, Paim JS, Vilasbôas AL. SUS: modelos assistenciais e vigilância da saúde. Informe epidemioloigico do SUS 1998; 7 (2): 7-28.

28. Rowe JW, Kahn RL. Successful aging. Gerontologist 1997; 37: 433-40. 